\title{
Selected published abstracts of Baylor researchers
}

\section{AMERICAN JOURNAL OF TRANSPLANTATION}

Belatacept-based immunosuppression in de novo liver transplant recipients: 1-year experience from a phase II randomized study

Klintmalm GB, Feng S, Lake JR, Vargas HE, Wekerle T, Agnes S, Brown KA, Nashan B, Rostaing L, Meadows-Shropshire S, Agarwal M, Harler MB, García-Valdecasas JC

Am J Transplant 2014 Jul 17 [Epub ahead of print]. Reprinted with permission from John Wiley \& Sons.

This exploratory phase II study evaluated the safety and efficacy of belatacept in de novo adult liver transplant recipients. Patients were randomized $(\mathrm{N}=260)$ to one of the following immunosuppressive regimens: (i) basiliximab + belatacept high dose [HD] + mycophenolate mofetil (MMF), (ii) belatacept $\mathrm{HD}+\mathrm{MMF}$, (iii) belatacept low dose [LD] + MMF, (iv) tacrolimus + MMF, or (v) tacrolimus alone. All received corticosteroids. Demographic characteristics were similar among groups. The proportion of patients who met the primary end point (composite of acute rejection, graft loss, death by month 6) was higher in the belatacept groups $(42 \%-48 \%)$ versus tacrolimus groups $(15 \%-38 \%)$, with the highest number of deaths and graft losses in the belatacept LD group. By month 12, the proportion surviving with a functioning graft was higher with tacrolimus + MMF (93\%) and lower with belatacept LD (67\%) versus other groups (90\%: basiliximab+ belatacept HD; 83\%: belatacept HD; 88\%: tacrolimus). Mean calculated GFR was $15-34 \mathrm{~mL} / \mathrm{min}$ higher in belatacept-treated patients at 1 year. Two cases of posttransplant lymphoproliferative disease and one case of progressive multifocal leukoencephalopathy occurred in belatacept-treated patients. Follow-up beyond month 12 revealed an increase in death and graft loss in another belatacept group (belatacept HD), after which the study was terminated.

\section{ANNALS OF VASCULAR SURGERY}

\section{The incidence and outcome of endothermal heat-induced thrombosis after endovenous laser ablation}

Kane K, Fisher T, Bennett M, Shutze W Jr, Hicks T, Grimsley B, Gable D, Pearl G, Smith B, Shutze W Sr

Ann Vasc Surg 2014 Jun 6 [Epub ahead of print]. Reprinted with permission from Elsevier.

Background: Endovenous laser ablation (EVLA) of the saphenous vein has become one of the preferred treatments for treating saphenous vein reflux that has resulted in symptomatic lower extremity venous insufficiency or varicose veins. This procedure was noted during initial reports to have a low incidence of postoperative thrombosis of the femoral or popliteal vein adjacent to the treated great saphenous vein (GSV) or small saphenous vein (SSV). Later clinical experience suggested that the actual incidence of this event is higher and it was subsequently termed endothermal heat-induced thrombosis (EHIT).

Methods: We reviewed the office records and the pre- and post-treatment ultrasounds of patients undergoing EVLA in our office from 2005 to 2010 to determine the frequency of EHIT in patients we had treated and then graded them according to a previously published classification.

Results: There were 528 veins treated in 192 men and 336 women. The clinical, etiology, anatomy, pathophysiology (CEAP) class for these patients was 1 (0), 2 (291), 3 (65), 4 (104), 5 (26), and 6 (40), respectively. The GSV was treated in 496 patients, the SSV in 22, and both were treated in 10 patients. EHIT occurred in 29 of the legs treated for an incidence of $5.1 \%$. The EHIT in the femoral vein were of level 3 (3), 4 (7), 5 (12), and 6 (3), respectively. Two patients developed EHIT in the popliteal vein after EVLA of the SSV. Treatment for the EHIT consisted of observation (13), anticoagulation (9), antiplatelet therapy (2), and nonsteroidal anti-inflammatory agents (1). Duration of therapy was usually 1 week, but 7 patients were treated for periods ranging from 1 to 7 weeks. No pulmonary emboli occurred in any of these patients. The EHIT resolved completely in all patients.

Conclusions: EHIT after EVLA occurs frequently and mainly consists of low-risk level 3, 4, and 5 deep vein thrombosis. The risk of pulmonary embolism is low and the EHIT typically resolves after 1 week. It can be treated with a short course of antiplatelet or anticoagulation therapy, although observation appears to be sufficient as well for lesser grades of EHIT.

\section{CLINICAL AND EXPERIMENTAL DERMATOLOGY}

Genetic markers of treatment response to tumour necrosis factor- $\alpha$ inhibitors in the treatment of psoriasis

Ryan C, Kelleher J, Fagan MF, Rogers S, Collins P, Barker JN, Allen M, Hagan R, Renfro L, Kirby B

Clin Exp Dermatol 2014;39(4):519-524. Reprinted with permission from John Wiley \& Sons.

Background: Anti-tumour necrosis factor (TNF)- $\alpha$ therapies have revolutionized the treatment of psoriasis; however, up to $50 \%$ of patients do not respond satisfactorily. Identification of pharmacogenetic markers of treatment response is an important step in the development of individually tailored treatment. The objective of this study was to assess the association of human leucocyte antigen (HLA)-C, killer immunoglobulin receptor (KIR) and vitamin D receptor (VDR) genotypes with response to treatment by etanercept and adalimumab.

Methods: This was a study of 138 patients with severe chronic plaque psoriasis who were treated with etanercept and/or adalimumab. Patients were classified as responders if they achieved a $75 \%$ reduction in PASI (PASI75) or were almost clear of psoriasis after 24 weeks of therapy. The frequencies of HLA-C and KIR haplotypes and VDR polymorphisms were compared in responders and nonresponders. The frequency of all HLA-C and KIR genotypes were compared between the 138 patients with psoriasis and 247 healthy donors.

Results: The number of patients classified as responders was 46 of 94 (49\%) in the etanercept group and 50 of $76(66 \%)$ in the adalimumab group. None of the HLA-C, KIR or VDR genotypes examined was predictive of treatment response. Compared with healthy controls, patients with psoriasis were more likely to have the HLA-C 06 genotype $(P<0.001)$ 
and less likely to have the HLA-C*07 genotype $(P<0.001)$, whereas there was no significant difference in frequencies of any KIR subtype.

Conclusions: Using the candidate gene approach to identify biomarkers of treatment response in psoriasis may have limited utility. This was a small study with limited power. Future larger studies are needed to further examine these findings and to explore alternative approaches to identify predictors of treatment response to biological agents.

\section{CURRENT CARDIOLOGY REPORTS}

\section{Ischemic and functional mitral regurgitation in heart failure: natural history and treatment}

Benjamin MM, Smith RL, Grayburn PA

Curr Cardiol Rep 2014;16(8):517. Reprinted with permission from Springer.

Functional mitral regurgitation (FMR) occurs when normal or nearly normal mitral leaflets are prevented from proper coaptation by underlying left ventricular (LV) dysfunction, mitral annular dilation, or both. FMR is associated with an adverse prognosis in nonischemic or ischemic LV dysfunction. Multiple studies have confirmed that even mild FMR portends a worse prognosis, and that the risk of FMR is independent of LV volumes and other clinical risk factors. FMR can be difficult to quantitate echocardiographically because it is load dependent and can vary considerably from exam to exam. There is a systematic tendency to underestimate FMR severity by echocardiography because the regurgitant orifice in FMR is typically elliptical, but the formula for calculating regurgitant orifice area assumes circular geometry. Treatment of FMR begins with guideline-directed medical therapy (GDMT) for LV dysfunction and heart failure, including cardiac resynchronization, if indicated. Revascularization should be considered for ischemic FMR, when indicated. Finally, mitral valve surgery should be considered in patients undergoing CABG in whom moderate or greater FMR is present, and also when severe symptomatic FMR persists despite optimal GDMT and revascularization. Percutaneous options for treatment of FMR are in development but are not currently approved in the US.

\section{INTERNATIONAL JOURNAL OF ENDOCRINOLOGY}

Inflammatory response in islet transplantation

Kanak MA, Takita M, Kunnathodi F, Lawrence MC, Levy MF, Naziruddin B

Int J Endocrinol 2014;2014:451035. Reprinted with permission.

Islet cell transplantation is a promising beta cell replacement therapy for patients with brittle type 1 diabetes as well as refractory chronic pancreatitis. Despite the vast advancements made in this field, challenges still remain in achieving high frequency and long-term successful transplant outcomes. Here we review recent advances in understanding the role of inflammation in islet transplantation and development of strategies to prevent damage to islets from inflammation. The inflammatory response associated with islets has been recognized as the primary cause of early damage to islets and graft loss after transplantation. Details on cell signaling pathways in islets triggered by cytokines and harmful inflammatory events during pancreas procurement, pancreas preservation, islet isolation, and islet infusion are presented. Robust control of pre- and peritransplant islet inflammation could improve posttransplant islet survival and in turn enhance the benefits of islet cell transplantation for patients who are insulin dependent. We discuss several potent anti-inflammatory strategies that show promise for improving islet engraftment. Further understanding of molecular mechanisms involved in the inflammatory response will provide the basis for developing potent therapeutic strategies for enhancing the quality and success of islet transplantation.

\section{JOURNAL OF THE AMERICAN COLLEGE OF SURGEONS}

\section{Compliance with recommended care at trauma centers: association with patient outcomes}

Shafi S, Barnes SA, Rayan N, Kudyakov R, Foreman M, Cryer HG, Alam HB, Hoff W, Holcomb J

J Am Coll Surg 2014;219(2):189-198. Reprinted with permission from Elsevier.

Background: State health departments and the American College of Surgeons focus on the availability of optimal resources to designate hospitals as trauma centers, with little emphasis on actual delivery of care. There is no systematic information on clinical practices at designated trauma centers. The objective of this study was to measure compliance with 22 commonly recommended clinical practices at trauma centers and its association with in-hospital mortality.

Study design: This retrospective observational study was conducted at 5 Level I trauma centers across the country. Participants were adult patients with moderate to severe injuries $(n=3,867)$. The association between compliance with 22 commonly recommended clinical practices and in-hospital mortality was measured after adjusting for patient demographics and injuries and their severity.

Results: Compliance with individual clinical practices ranged from as low as $12 \%$ to as high as $94 \%$. After adjusting for patient demographics and injury severity, each $10 \%$ increase in compliance with recommended care was associated with a $14 \%$ reduction in the risk of death. Patients who received all recommended care were 58\% less likely to die (odds ratio $=0.42 ; 95 \% \mathrm{CI}, 0.28-0.62$ ) compared with those who did not.

Conclusions: Compliance with commonly recommended clinical practices remains suboptimal at designated trauma centers. Improved adoption of these practices can reduce mortality.

\section{JOURNAL OF NURSING ADMINISTRATION}

\section{Development of a tool to measure user experience following electronic health record implementation}

Xiao Y, Montgomery DC, Philpot LM, Barnes SA, Compton J, Kennerly D

J Nurs Adm 2014;44(7/8):423-428. Reprinted with permission from Wolters Kluwer Health.

Objective: The aim of this study was to develop a survey tool to assess electronic health record (EHR) implementation to guide improvement initiatives.

Background: Survey tools are needed for ongoing improvement and have not been developed for aspects of EHR implementation.

Methods: The Baylor EHR User Experience (UX) survey was developed to capture 5 concept domains: training and competency, usability, infrastructure, usefulness, and end-user support. Validation efforts included content validity assessment, a pilot study, and analysis of 606 nurse respondents. The revised tool was sent to randomly sampled EHR nurse-users in 11 acute care facilities.

Results: A total of 1,301 nurses responded (37\%). Internal consistency of the survey tool was excellent (Cronbach's $\alpha=.892$ ). Survey responses including 1,819 open comments were used to identify and prioritize improvement efforts in areas such as education, support, optimization of EHR functions, and vendor change requests. 
Conclusion: The Baylor EHR UX survey was a valid tool that can be useful for prioritizing improvement efforts in relation to EHR implementation.

\section{The impact of opening visitation access on patient and family experience}

Nuss T, Kelly KM, Campbell KR, Pierce C, Entzminger JK, Blair BK, Wissinger L, Bryant L, Walker JL.

J Nurs Adm 2014;44(7/8):403-410. Reprinted with permission from Wolters Kluwer Health.

Restrictive visiting hours have been an obstacle to family participation in care. To support increased and consistent access to patients, Baylor Health Care System implemented a system-wide approach to open access for visitation across all facilities. Nursing and medical leadership led the communication efforts, and shared nursing governance guided revisions to existing policies. Data collected from 13 hospitals demonstrated that patients and families felt more informed; that the nursing staff were more courteous and respectful and explained things in a way that could be understood; that the staff attitude toward visitors was markedly improved; and that comfort and accommodations for guests were extended and improved. The resources needed to deploy these changes are outlined as well as the iterative process needed to create a positive impact on the family partnership in care.

\section{LUNG CANCER}

BRAFV600E-mutated lung adenocarcinoma with metastases to the brain responding to treatment with vemurafenib

Robinson SD, O'Shaughnessy JA, Cowey CL, Konduri K

Lung Cancer 2014;85(2):326-330. Reprinted with permission from Elsevier.

Somatic $B R A F$ mutations have been reported in $1-4 \%$ of non-small cell lung cancer (NSCLC), primarily in adenocarcinomas with the $B R A F(\mathrm{~V} 600 \mathrm{E})$ mutation in about $50 \%$ of the cases. The role of $B R A F$ mutation in NSCLC and the treatment for tumors with such mutations is still evolving. Our patient had metastatic NSCLC with metastases to her brain. Due to the BRAF (V600E) mutation in her tumor and her poor functional status, we offered her off-label treatment with vemurafenib, a BRAF inhibitor approved for use in metastatic melanoma. Our patient's visceral disease improved, supporting vemurafenib's efficacy in the treatment of metastatic $B R A F$-mutated NSCLC. The regression of intracranial disease indicated vemurafenib was able to cross the blood-brain barrier and was efficacious in treating brain metastases in this patient with lung cancer.

\section{MEDICINE}

Morphologic features of the recipient heart in patients having cardiac transplantation and analysis of the congruence or incongruence between the clinical and morphologic diagnoses

Roberts WC, Roberts CC, Ko JM, Filardo G, Capehart JE, Hall SA

Medicine 2014;93(5):211-235. Reprinted with permission from Wolters Kluwer Health.
Cardiac transplantation (CT) has been one of the great medical advances of the last nearly 50 years. We studied the explanted hearts of 314 patients having CT at Baylor University Medical Center Dallas from 1993 to 2012, and compared the morphologic diagnoses to the clinical diagnoses before CT. Among the 314 patients the morphologic and clinical diagnoses were congruent in $272(87 \%)$ and incongruent in $42(13 \%)$. Most of the incongruity occurred among the 166 patients with non-ischemic cardiomyopathy (non-IC) (36/166 [22\%]), and of that group the major incongruity occurred among the patients with hypertrophic cardiomyopathy (7/17 [41\%]), non-compaction left ventricular cardiomyopathy (NCLVC) (3/3 [100\%]), mononuclear myocarditis (3/3 [100\%]), arrhythmogenic right ventricular cardiomyopathy (ARVC) $(4 / 4$ [100\%]), and cardiac sarcoidosis $(8 / 8$ [100\%]). The phrase "non-IC" is a general term that includes several subsets of cardiac diseases and simply means "insignificant narrowing of 1 or more of the epicardial coronary arteries," but it does not specify the specific cause of the heart failure leading to CT. A number of cardiac illustrations are provided to demonstrate the morphologic variability occurring among the patients with IC and non-IC.

\section{PLOS ONE}

\section{MicroRNA miR-J1-5p as a potential biomarker for JC virus infection in the gastrointestinal tract}

Link A, Balaguer F, Nagasaka T, Boland CR, Goel A

PLoS One 2014;9(6):e100036. Reprinted with permission.

Introduction: JC virus (JCV), a human polyomavirus that causes progressive multifocal leukoencephalopathy (PML), has been linked to colorectal cancer (CRC). However, determination of JCV infection and its role in carcinogenesis has been challenging, highlighting the need for better diagnostic strategies for this virus. JCV-specific microRNAs (miRNAs) were identified and shown to negatively regulate oncogenic JCV T-Ag. Herein, we determined the pattern of JCV miRNA expression in clinical specimens from healthy subjects and CRC patients.

Material and methods: JCV miRNA expression was validated in CRC cell lines transfected with the JCV T-Ag. Results were confirmed using CRC tissues that expressed T-Ag. Expression of JCV-specific miR-J1$5 \mathrm{p}$ was measured in fresh stool samples from healthy volunteers, and samples from fecal occult blood test kits from healthy subjects and patients with colorectal neoplasms.

Results: JCV miR-J1-5p was detected in JCV-transfected, but not vector-transfected, CRC cells, and was stable between cell passages. MiR-J1-5p was present in all six JCV T-Ag+ CRC samples. Surprisingly, JCV miRNA was detectable in all normal tissues, but the expression was much lower in CRC tissues. Similarly, miR-J1-5p expression was present in all fecal samples, but expression was lower in CRCs compared to controls or adenoma patients.

Conclusion: JC virus-specific miR-J1-5p miRNA is a potential biomarker for viral infection, and the lower expression in patients with colonic neoplasia highlights its biological role regulating oncogenic $\mathrm{T}-\mathrm{Ag}$ expression in CRC.

Impact: JCV-specific miRNA is a candidate for the development of a non-invasive screening test, as well as therapeutic intervention for JCV-associated diseases.

If you are a Baylor researcher and would like your published abstract to be included in this section, please e-mail the PubMed citation to Cynthia. Orticio@BaylorHealth.edu. 